\title{
RSS-based DoA Estimation Using ESPAR Antenna Radiation Patterns Spline Interpolation
}

\author{
M. Groth ${ }^{1}$, K. Nyka ${ }^{2}$, Member, IEEE, and L. Kulas ${ }^{3}$, Senior Member, IEEE \\ Department of Microwave and Antenna Engineering \\ Faculty of Electronics, Telecommunications and Informatics, Gdansk University of Technology, \\ Gdansk, Poland \\ ${ }^{1}$ mateusz.groth@eti.pg.gda.pl, ${ }^{2}$ krzysztof.nyka@eti.pg.gda.pl, ${ }^{3}$ lukasz.kulas@eti.pg.gda.pl
}

\begin{abstract}
In this paper, it is shown how power pattern crosscorrelation (PPCC) algorithm, which relies on received signal strength (RSS) values recorded at electronically steerable parasitic array radiator (ESPAR) antenna output port, used for directionof-arrival (DoA) estimation, can easily be improved by applying spline interpolation to radiation patterns recorded in the calibration phase of the DoA estimation process. The proposed method allows one to measure ESPAR antenna's radiation patterns during the initial calibration phase with much coarser angular resolution than required for linearly interpolated radiation patterns. Simulation results indicate that the overall DoA estimation accuracy can be kept at the similar level even for a few number of points, which, when applied in anechoic chamber calibration procedure of wireless sensor network (WSN) nodes equipped with ESPAR antennas, will have a noticeable influence on the overall calibration time and therefore also on deployment costs in practical WSN applications.
\end{abstract}

Keywords-Switched-beam antenna, ESPAR antenna, directionof-arrival (DoA), received signal strength (RSS), interpolation algorithm.

\section{INTRODUCTION}

During the recent years, a vast range of foreseen applications of indoor positioning influenced on the techniques of determining the position and obtaining the necessary input information for positioning algorithms. Most common methods are based on the measured RSS values, obtained by the power sensor of wireless device. However due to the characteristics of indoor propagation environment, the information, mostly employed for multilateration algorithms as a range equivalent, might be significantly flawed [13]. The other use of RSS is to estimate the direction of arrival of the signal impinging the ESPAR antenna. In this solution, the maximum likelihood criteria is used to determine the most probable direction from which the signal arrives to the antenna. Then the information is processed using the triangulation algorithm [14], [15].

Direction-of-arrival (DoA) is an important technique also frequently used for indoor positioning and to enhance capabilities of modern wireless communication systems [1]. Often, it involves digital beamforming, for which a series of digital signal processing (DSP) units is necessary [2], which highly increase the overall cost of the system. Alternatively, electronically steerable parasitic array radiator (ESPAR) antenna can be used [3]-[5], which consists of only one singleport output connected to the active element surrounded by a number of parasitic elements. By adjusting the reactance values

Research leading to these results has received funding from the EU ECSEL Joint Undertaking under grant agreement no. 737459 (project Productive4.0) and from the National Centre for Research and Development on behalf of the Ministry of Science and Higher Education in Poland. connected to the parasitic elements, it is possible to shape antenna's radiation pattern. Such a property of ESPAR antennas, together with a proper DoA algorithm, such as MUltiple SIgnal Classification algorithm (MUSIC) [6] or power pattern crosscorrelation algorithm (PPCC) [4], gives the opportunity to estimate the direction of arrival of the signal impinging the antenna.

In wireless sensor network (WSN) applications, DoA estimation is often utilized to improve parameters or functionality of the system, such as coverage, energy-efficiency, localization and connectivity [7]-[9]. The PPCC algorithm has been already implemented in WSN applications [10], but the main disadvantage of the solution is the necessity of performing a time consuming calibration of each node of the system. To obtain accurate DoA estimation, ESPAR radiation patterns need to be measured with at least $1^{\circ}$ resolution [4], [10] what leads to time and cost consuming deployment of such system. As a solution to this drawback, PPCC-based DoA algorithm for ESPAR antenna which uses linearly interpolated radiation patterns has been introduced in [11]. The method allows to shorten the calibration procedure of the DoA algorithm by measuring ESPAR antenna's radiation patterns with coarser angular step $\Delta \varphi$, which is then linearly interpolated. The main weakness of this method is that, with angular steps larger than $12^{\circ}$, the DoA estimation error significantly grows [11].

In this paper, a modification to the PPCC-based DoA algorithm relying on interpolated ESPAR antenna radiation patterns [11] is proposed and verified in numerical simulations. The proposed improvement uses circular cubic spline to obtain ESPAR antenna radiation patterns from very coarse measurements. Numerical simulations results indicate that the proposed concept improves the accuracy of RSS-based DoA estimation giving the opportunity to further reduce time needed for the calibration procedure, while keeping the precision of the estimation at the similar level.

\section{ESPAR ANTENNA DESIGN}

The proposed DoA estimation method is based on ESPAR antenna design with 12 passive elements and one active monopole located in the center of the ground plane. Results provided in [4] indicate that the best DoA estimation can be obtained for the narrowest directional main beams having high front-to-back ratios. In selected design, $1.7 \mathrm{~mm}$ FR4 laminate with top-layer metallization has been used. Passive elements can be connected to the ground or opened by a dedicated switch, connected to the ground plane end of the element [4]. This way 
every parasitic element becomes a reflector when connected to the ground and a director when opened. Such a design gives the possibility of controlling the antenna's radiation pattern electronically. As defined in [10], each antenna configuration can be described by the steering vector $V=$ $\left[v_{1}, v_{2}, \ldots, v_{12}\right]$ where $v_{n}$ describes the current state of the $n$th parasitic element and can take values $v_{n}=0$ when it is shortened to the ground or $v_{n}=1$ when opened.

The proposed ESPAR antenna, shown in Fig.1, has been designed and simulated in FEKO electromagnetic simulation software at the center frequency of $2.484 \mathrm{GHz}$. The antenna design re-used initial dimensions proposed in [10] and has been optimized to provide radiation patterns, which representative is visible in Fig. 2, that can be utilized in DoA estimations [11].

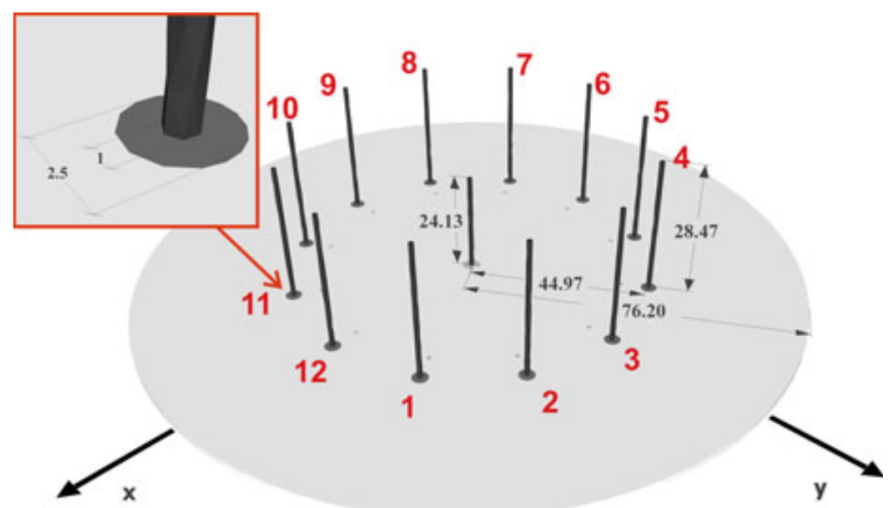

Fig. 1. Designed ESPAR antenna together with numbering of its parasitic elements. All the dimensions are in millimeters.

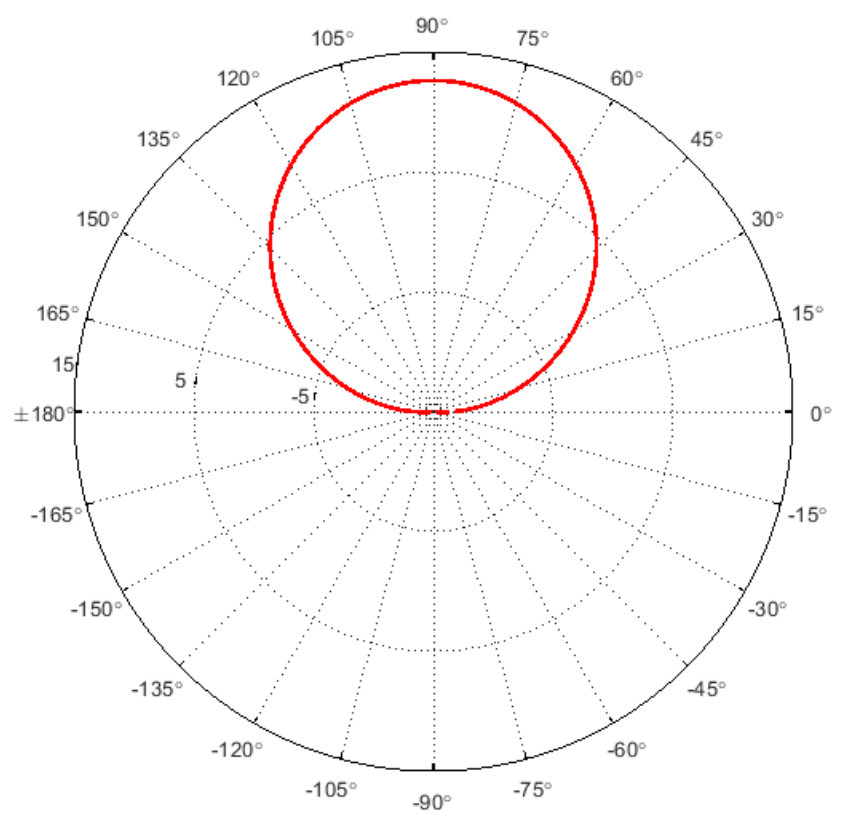

Fig. 2. Designed ESPAR antenna's horizontal plane radiation pattern (in $\mathrm{dBi}$ ). Main beam's direction $90^{\circ}$ is aligned with y axis and has been obtained for the steering vector $[1,1,1,1,1,0,0,0,0,0,0,0]$ (see text for explanations).

\section{DIRECTION-OF-ARRIVAL ESTIMATION USING RADIATION PATTERNS SPLINE INTERPOLATION}

The proposed DoA estimation is based on RSS-based PPCC algorithm [4], which calculates cross-correlation coefficient between each of antenna outputs recorded for all main beam directions and measured antenna's radiation pattern. As presented in [11], for the discreet values of antenna's radiation patterns measured with the angular step $\Delta \varphi$, the crosscorrelation coefficient can be written in its discreet form:

$$
g=\frac{\sum_{n=1}^{12}\left(p^{n} Y\left(V_{\max }^{n}\right)\right)}{\sqrt{\sum_{n=1}^{12}\left(p^{n} p^{n}\right)} \sqrt{\sum_{n=1}^{12} Y\left(V_{\max }^{n}\right)^{2}}}
$$

where "o" is the Hadamard product, $g=$ $\left[\Gamma\left(\varphi_{1}\right), \Gamma\left(\varphi_{2}\right), \ldots, \Gamma\left(\varphi_{I}\right)\right]^{T}$ is a vector of discretized values of correlation coefficients $\Gamma(\varphi)$ for values $\varphi=\left[\varphi_{1}, \varphi_{2}, \ldots, \varphi_{I}\right]^{T}$, $\boldsymbol{p}^{n}=\left[p_{1}^{n}, p_{2}^{n}, \ldots, p_{I}^{n}\right]^{T}$ is a discreet representation of ESPAR antenna's radiation pattern values for all corresponding steering vectors measured in an anechoic chamber with the angular step precision $\Delta \varphi,\left\{Y\left(V_{\text {max }}^{1}\right), Y\left(V_{\text {max }}^{2}\right), \ldots, Y\left(V_{\text {max }}^{12}\right)\right\}$ are output power values recorded during the actual DoA estimation process for a signal impinging the antenna from an unknown direction. In the abovementioned formula, it is easy to notice that by decreasing the angular step $\Delta \varphi$, the precision of $\Gamma(\varphi)$ increase. This implies the conclusion that for more accurate DoA estimations the longer calibration procedure is required, which influences the final WSN-based DoA system deployment time.

To reduce the potential complexity of the calibration procedure, linear interpolation of antenna radiation patterns have been introduced [11]. This gives an opportunity to reduce the number of calibration points necessary to be measured and still maintain satisfactory precision of PPCC-based DoA estimation. In [11], it has been shown that it is possible to obtain similarly high precision of DoA estimation using only 15 calibration points instead of 360 . In this paper, cubic spline interpolation of ESPAR antenna radiation patterns is introduced as a way of further enhancement of the method proposed in [11].

The main drawback of linear interpolation of the antenna radiation patterns is that the fitting results deteriorate for larger angular steps $\Delta \varphi$. It is expected, that exchanging linear interpolation functions with cubic polynomials will improve the interpolation effects, and as a result, will provide better DoA estimation results comparing to those with linear interpolation.

The cubic spline $S(\varphi)$ function can be described as:

$$
S(\varphi)=\left\{\begin{array}{c}
C_{1}(\varphi), \varphi_{0} \leq \varphi \leq \varphi_{1} \\
C_{2}(\varphi), \varphi_{1}<\varphi \leq \varphi_{2} \\
\ldots \\
C_{n}(\varphi), \varphi_{n-1}<\varphi \leq \varphi_{n}
\end{array}\right.
$$

where $C_{i}=a_{i}+b_{i} \varphi+c_{i} \varphi^{2}+d_{i} \varphi^{3}$ is a cubic function of subinterval $\left[\varphi_{i-1}, \varphi_{i}\right], i=1, \ldots, n$. To determine the cubic spline, coefficients $a_{i}, b_{i}, c_{i}, d_{i}$ need to be found using the boundary conditions presented in (3): 
2018 International Conference on Indoor Positioning and Indoor Navigation (IPIN), 24-27 September 2018, Nantes, France

$$
\begin{aligned}
& C_{i}\left(\varphi_{i-1}\right)=P_{i-1}, \quad i=1, \ldots, n \\
& C_{i}\left(\varphi_{i}\right)=P_{i}, \quad i=1, \ldots, n \\
& C_{i}^{\prime}\left(\varphi_{i}\right)=C_{i+1}^{\prime}\left(\varphi_{i}\right), \quad i=1, \ldots, n-1 \\
& C_{i}^{\prime \prime}\left(\varphi_{i}\right)=C_{i+1}^{\prime \prime}\left(\varphi_{i}\right), \quad i=1, \ldots, n-1
\end{aligned}
$$

Since the antenna radiation pattern characteristics is periodic with period $p=2 \pi$, additional conditions for the periodic function have to be taken into account:

$$
\begin{aligned}
C_{1}\left(\varphi_{0}\right) & =C_{n}\left(\varphi_{n}\right) \\
C_{1}^{\prime}\left(\varphi_{0}\right) & =C_{n}^{\prime}\left(\varphi_{n}\right) \\
C_{1}^{\prime \prime}\left(\varphi_{0}\right) & =C_{n}^{\prime \prime}\left(\varphi_{n}\right)
\end{aligned}
$$

As a result, a set of cubic functions for determined intervals can be obtained.

\section{NUMERICAL SIMULATIONS}

In order to verify the presented concept and the overall accuracy improvement using spline interpolation, the designed ESPAR antenna has been simulated in FEKO electromagnetic simulation software to obtain antenna radiation patterns for every of the 12 main beam directions at $2.484 \mathrm{GHz}$. Obtained characteristics have been sampled with a set of different angular steps precisions. Then, for each $\Delta \varphi$ value the $1^{\circ}$ step radiation patterns have been recreated using linear and spline interpolation. The results presented in Fig. 3, Fig. 4 and Table I show that spline interpolation is more accurate than linear one.

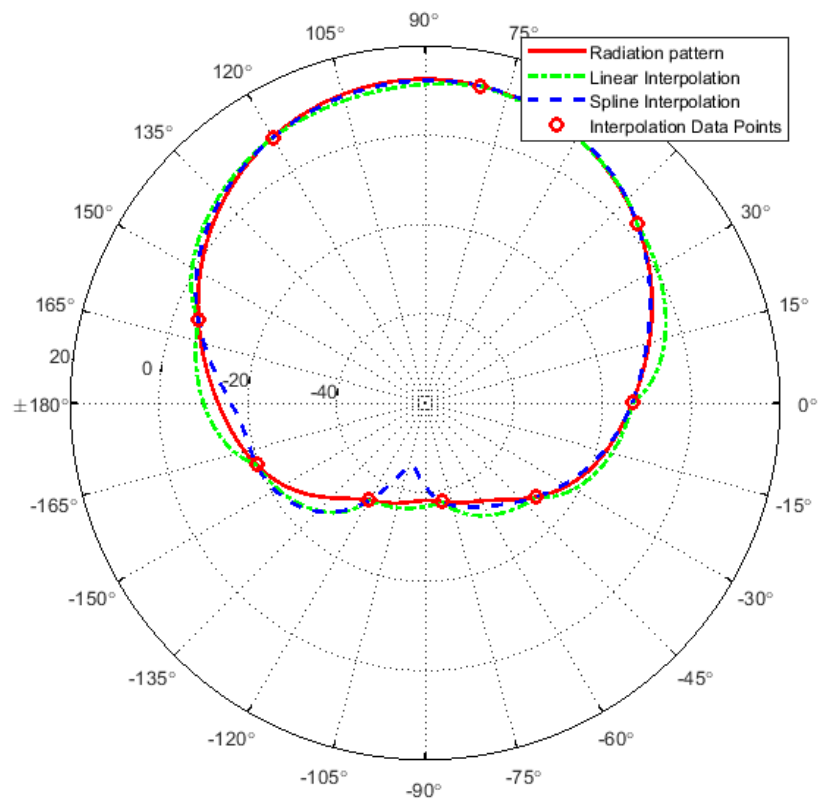

Fig. 3. Comparison of interpolated antenna radiation pattern characteristics (in $\mathrm{dBi}$ ) for $\Delta \varphi=40^{\circ}$ in polar representation.

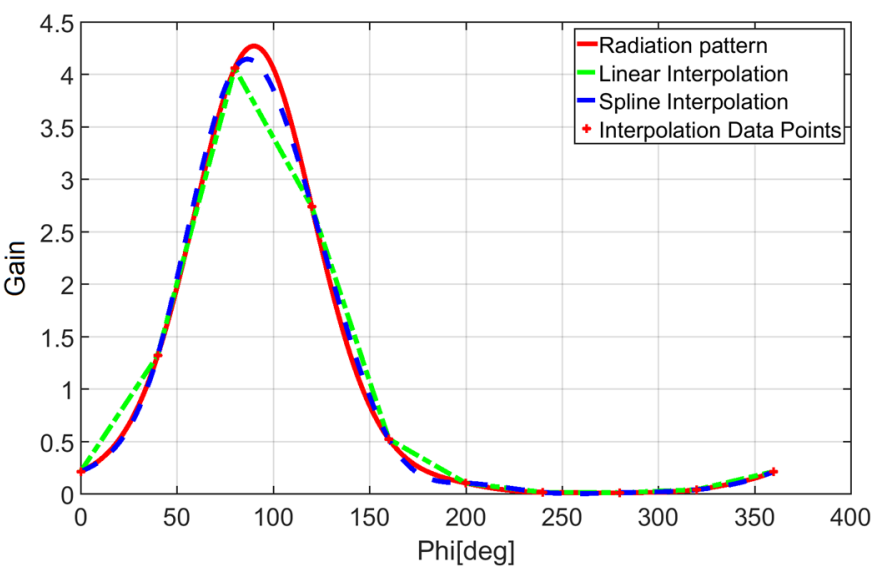

Fig. 4. Comparison of interpolated antenna radiation pattern characteristics for $\Delta \varphi=40^{\circ}$ in linear representation.

TABLE I. ANTENNA RADIATION PATTERN RMS INTERPOLATION ERROR

\begin{tabular}{|c|c|c|c|c|c|c|c|c|c|}
\hline & $\Delta \boldsymbol{\varphi}=$ & $\mathbf{3}^{\circ}$ & $\mathbf{6}^{\circ}$ & $\mathbf{1 8}^{\circ}$ & $\mathbf{2 4}^{\circ}$ & $\mathbf{3 6}^{\circ}$ & $\mathbf{4 0}^{\circ}$ & $\mathbf{4 5}^{\circ}$ & $\mathbf{6 0}^{\circ}$ \\
\hline $\begin{array}{l}\text { Gain } \\
\text { RMS } \\
\text { error }\end{array}$ & $\begin{array}{c}\text { Linear } \\
\text { interpolation }\end{array}$ & 0.0012 & 0.0046 & 0.041 & 0.0719 & 0.1629 & 0.1884 & 0.1859 & 0.5125 \\
$\begin{array}{c}\text { Spline } \\
\text { interpolation }\end{array}$ & $6 \cdot 10^{-7}$ & $10^{-5}$ & 0.0013 & 0.0058 & 0.0574 & 0.0691 & 0.0125 & 0.3927 \\
\hline
\end{tabular}

In next step, the obtained sets of antenna radiation patterns have been used in DoA simulations in MATLAB. To this end, the power of a test signal impinging the antenna has been set to $10 \mathrm{dBm}$, and for every considered direction 10 snapshots were generated. Additionally, to achieve more realistic results, additive white Gaussian noise has been added to introduce a specific signal-to-noise ratio (SNR) for every test.

To determine DoA estimation errors, the difference between real and estimated position has been calculated for each of the horizontal directions $\varphi_{t}$, which were determined by setting the transmitting antenna position with a $5^{\circ}$ angular step around the receiving antenna. The results for $\mathrm{SNR}=10 \mathrm{~dB}$ and $\Delta \varphi=90^{\circ}$ for both interpolations are shown in Fig. 5. The comparison of the results for different values of SNR are presented in Fig. 6 and Table II. Additionally, root-mean-square (RMS) error values have been calculated and compared for linear and spline interpolation of antenna radiation characteristics, and presented in Fig. 7 and Table III, which also contains calibration time for different $\Delta \varphi$ measured for our test rotation table. The calibration time can be described as below:

$$
t_{c}=12 \cdot\left[\left(1-\frac{\Delta \varphi}{360}\right) \cdot t_{r}+\left(\frac{360}{\Delta \varphi}\right) \cdot t_{m}\right]
$$

where $t_{r}$ is the time of full rotation of the antenna from $\varphi_{0}=$ $0^{\circ}$ to $\varphi_{n}=360^{\circ}$ and $t_{m}$ is the time of measurement at each direction $\varphi$.

The results indicate that the implementation of spline interpolation of ESPAR antenna radiation patterns provides lower DoA estimation errors than when linear interpolation is used. This leads to the conclusion that the angular step $\Delta \varphi$ can be increased even more, reducing the calibration time necessary to measure all ESPAR radiation patterns. 


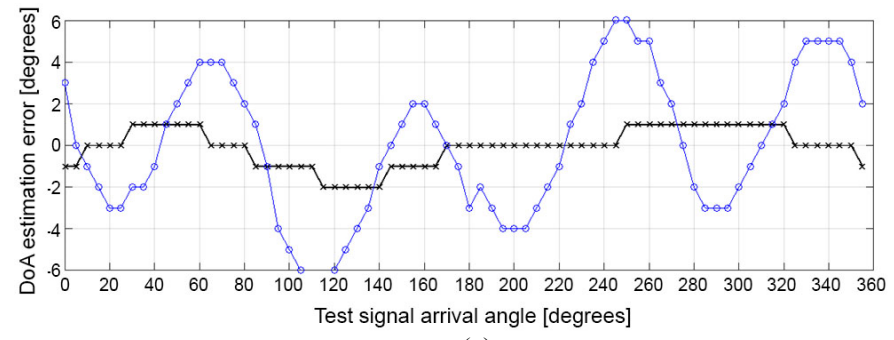

(a)

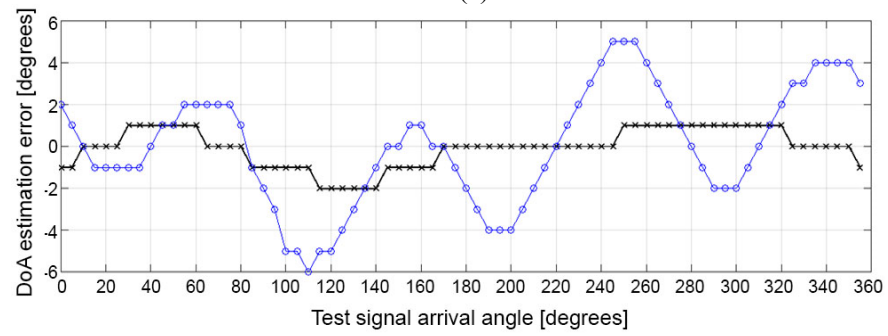

(b)

Fig. 5. DoA estimation error obtained from measurements at $\mathrm{SNR}=10 \mathrm{~dB}$ for different angular step precisions used during calibration phase. The thick black line with ' $x$ ' markers represents fine $1^{\circ}$ resolution used in calibration phase, while the thin one with circle markers represents DoA estimation errors for $\Delta \varphi=90^{\circ}$ resolution used in fast calibration procedure with (a) linear interpolation and (b) periodic spline interpolation.

It is clearly visible that the larger angular step $\Delta \varphi$ the stronger improvement over DoA estimation results generated using linearly interpolated ESPAR antenna radiation patterns can be obtained. While for the $\Delta \varphi$ values lower than $40^{\circ}$ the results are similar for both types of interpolation, for higher values the improvement is easily noticeable. For $\Delta \varphi=90^{\circ}$, which is 4 calibration points for each of the ESPAR antenna radiation patterns, the RMS value achieves reduction from $3.41^{\circ}$ for linear interpolation to $2.73^{\circ}$ for spline interpolation which gives an improvement of about $20 \%$. This leads to the reduction of the deployment time of localization system giving the possibility of using less calibration points for each localization node. Since this applies to every node, the larger system, the more significant time reduction can be achieved.

Using the Intel Core i7 $2.6 \mathrm{GHz}$ laptop, the spline interpolation of the radiation patterns using the MATLAB Curve Fitting Toolbox functions took $4.65 \mathrm{~ms}$ for $\Delta \varphi=45^{\circ}$. This is about 3.2 times longer than for the linear interpolation, but still negligibly short in terms of the DoA estimation calibration procedure, which takes almost half an hour for a single WSN node [11]. In consequence, as results in Table III indicate, the proposed calibration procedure, can either improve the overall DoA estimation accuracy for larger angular step $\Delta \varphi$ or allows for further reduction of the calibration time. It needs to be mentioned that in our testbed time of rotation $t_{r}$ is much longer than time of taking the measurement for particular rotation $t_{m}$. One can notice, however, that for DoA-calibration designated testbeds with larger $t_{m}: t_{r}$ relations, more significant reduction of the calibration time can be obtained. To verify that, calibration time for a testbed with NI ST23-4 stepper motor together with NI SMD-7615 drive has been measured. The device can achieve resolution up to 20.000 steps per single $360^{\circ}$ revolution and include anti-resonance and torque ripple smoothing features. The results presented in Table IV show that in DoA specific calibration set the calibration time increases proportionally with the number of calibration points. This is especially important for calibration of large systems where potential reduction of calibration time multiplies by the number of nodes with DoA estimation functionality where multiple calibration planes for each node have been used [12].

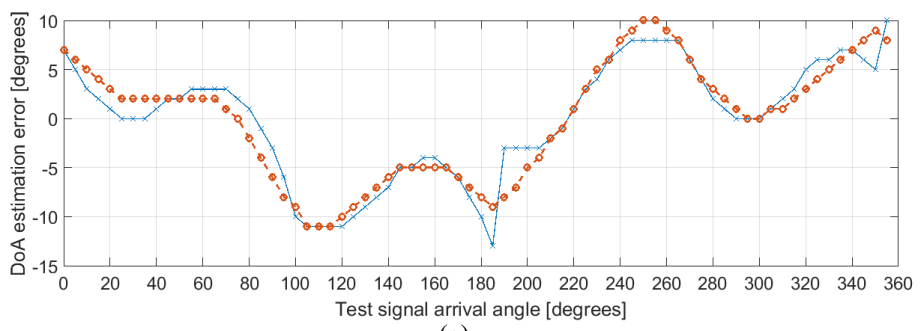

(a)

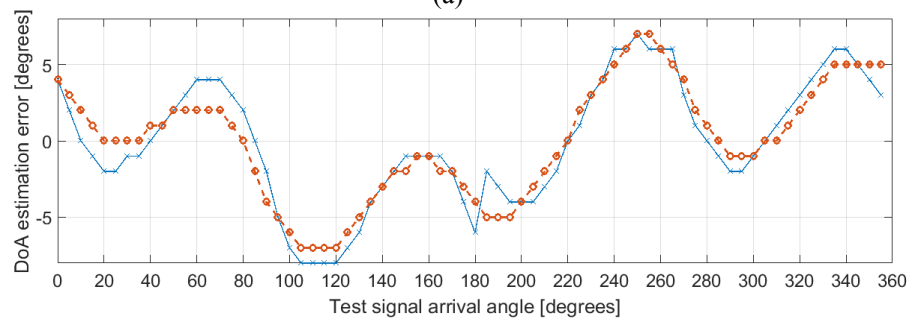

(b)

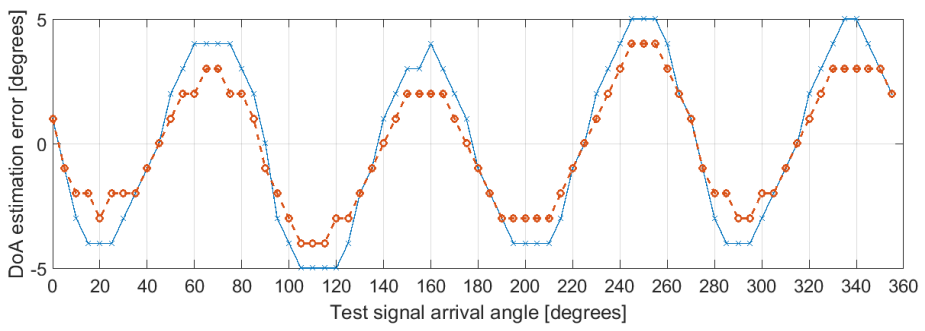

(c)

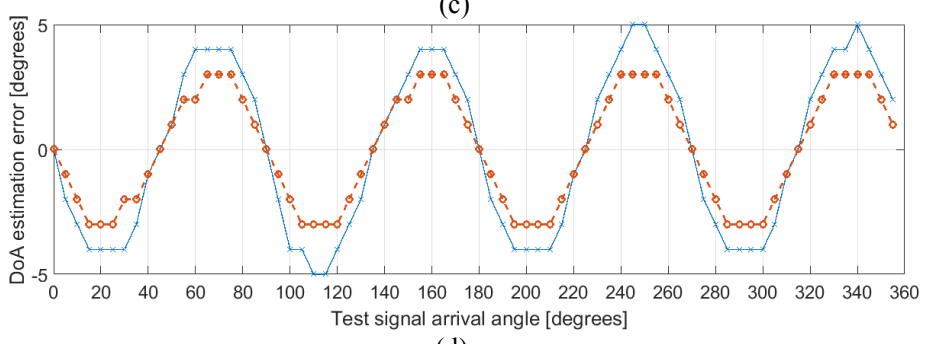

(d)

Fig. 6. DoA estimation error obtained from measurements at (a) $\mathrm{SNR}=0 \mathrm{~dB}$, (b) $\mathrm{SNR}=5 \mathrm{~dB}$, (c) $\mathrm{SNR}=20 \mathrm{~dB}$ and (d) $\mathrm{SNR}=30 \mathrm{~dB}$ for $\Delta \varphi=90^{\circ}$. The thick red line with circle markers represents spline interpolation, while the thin one with ' $\mathrm{x}$ ' markers represents linear interpolation.

TABLE II. RMS INTERPOLATION ERROR FOR $\Delta \varphi=90^{\circ}$ AND DIFFERENT SNR VALUES

\begin{tabular}{|c|c|c|c|c|c|}
\hline SNR [dB] & $\mathbf{0}$ & $\mathbf{5}$ & $\mathbf{1 0}$ & $\mathbf{2 0}$ & $\mathbf{3 0}$ \\
\hline $\begin{array}{c}\text { Linear } \\
\text { interpolation }\end{array}$ & 5.8178 & 3.9791 & 3.4142 & 3.2382 & 3.2167 \\
\hline Spline interpolation & 5.9594 & 3.7118 & 2.7332 & 2.3333 & 2.2142 \\
\hline
\end{tabular}




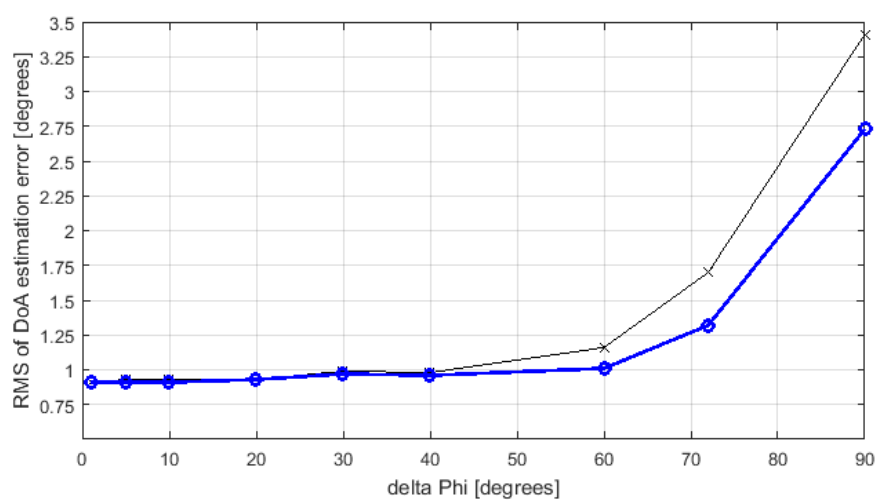

Fig. 7. DoA estimation error obtained from measurements at $\mathrm{SNR}=10 \mathrm{~dB}$ as a function of angular step precision $\Delta \varphi$ used during calibration phase. The thick line represents RMS of DoA estimation error using spline interpolation for antenna radiation pattern estimation, while the thin line represents RMS of DoA estimation error using linear interpolation.

TABLE III. ROOT-MEAN-SQUARE DOA ESTIMATION ERROR

\begin{tabular}{|c|c|c|c|c|c|c|c|c|c|c|c|}
\hline & \multicolumn{9}{|c|}{ Angular resolution $\Delta \varphi$} \\
\hline & & & $1^{\circ}$ & $5^{\circ}$ & $10^{\circ}$ & $20^{\circ}$ & $30^{\circ}$ & $40^{\circ}$ & $60^{\circ}$ & $72^{\circ}$ & $90^{\circ}$ \\
\hline \multicolumn{3}{|c|}{$\begin{array}{l}\text { Calibration } \\
\text { time }[\mathrm{mm}: \mathrm{ss}]\end{array}$} & $33: 05.8$ & $19: 12$ & $17: 22.6$ & $16: 25.2$ & $16: 12$ & $16: 01.2$ & $15: 49.2$ & $15: 48$ & $15: 46.6$ \\
\hline \multirow{4}{*}{ 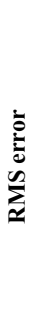 } & \multirow{2}{*}{ 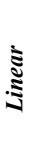 } & $\begin{array}{c}\text { Absolute } \\
{\left[^{\circ}\right]}\end{array}$ & 0.91 & 0.93 & 0.93 & 0.93 & 0.99 & 0.98 & 1.16 & 1.70 & 3.41 \\
\hline & & $\begin{array}{c}\text { Change } \\
{[\%]}\end{array}$ & 100 & 102 & 101 & 102 & 108 & 107 & 127 & 187 & 373 \\
\hline & \multirow{2}{*}{ है } & $\begin{array}{c}\text { Absolute } \\
{\left[{ }^{\circ}\right]}\end{array}$ & 0.91 & 0.91 & 0.91 & 0.93 & 0.97 & 0.96 & 1.01 & 1.32 & 2.73 \\
\hline & & $\begin{array}{c}\text { Change } \\
{[\%]}\end{array}$ & 100 & 100 & 100 & 102 & 106 & 105 & 111 & 145 & 299 \\
\hline
\end{tabular}

TABLE IV. CALibration TIME FOR DifFERENT ANGUlar Resolution USING NI ST23-4 STEPPER MOTOR

\begin{tabular}{|c|c|c|c|c|c|c|c|c|c|}
\hline & \multicolumn{7}{|c|}{ Angular resolution $\Delta \varphi$} \\
\cline { 2 - 8 } & $1^{\circ}$ & $5^{\circ}$ & $10^{\circ}$ & $20^{\circ}$ & $30^{\circ}$ & $40^{\circ}$ & $60^{\circ}$ & $72^{\circ}$ & $90^{\circ}$ \\
\hline $\begin{array}{c}\text { Calibration } \\
\text { time [mm:ss] }\end{array}$ & $16: 12$ & $03: 10.6$ & $01: 37.2$ & $00: 52.8$ & $00: 33$ & $00: 23.4$ & $00: 17.4$ & $00: 14$ & $00: 11.4$ \\
\hline
\end{tabular}

\section{CONCLUSIONS}

In this paper, it has been presented, how PPCC DoA estimation algorithm can be further improved by employing circular spline interpolation for radiation patterns interpolation. Presented method allows one to shorten the time needed for the deployment of WSN-based indoor positioning system nodes equipped with ESPAR antennas by giving the possibility to measure ESPAR antenna radiation patterns with large angular step resolution. In consequence, shorter anechoic chamber measurements during the PPCC algorithm calibration phase can be obtained. Numerical simulations indicate that it is possible to obtain lower error values of DoA estimations comparing to those where linear interpolation was used. Thus, for circular spline interpolation, larger values of calibration angular step $\Delta \varphi$ can be taken under consideration to further shorten the calibration time maintaining the required DoA estimation accuracy.

\section{ACKNOWLEDGMENT}

The authors would like to thank the Academic Computer Centre in Gdansk, Poland (TASK) were all the calculations were carried out.

The authors would like to thank the anonymous reviewers for their helpful and constructive comments that greatly contributed to improving the final version of the paper.

\section{REFERENCES}

[1] A. Gaber and A. Omar, "A Study of Wireless Indoor Positioning Based on Joint TDOA and DOA Estimation Using 2-D Matrix Pencil Algorithms and IEEE 802.11ac," in IEEE Transactions on Wireless Communications, vol. 14, no. 5, pp. 2440-2454, May 2015.

[2] D. H. Johnson and D. E. Dudgeon, Array Signal Processing, Concepts and Techniques, Englewood Cliffs, NJ: Prentice-Hall, 1993.

[3] Sathish Chandran, Advances in direction-of-arrival estimation, Artech House, London, 2005.

[4] E. Taillefer, A. Hirata and T. Ohira, "Direction-of-arrival estimation using radiation power pattern with an ESPAR antenna," IEEE Transactions on Antennas and Propagation, vol. 53, no. 2, pp.678-684, Feb. 2005.

[5] M. Rzymowski, K. Nyka, L. Kulas, "Enhancing Performance of Switched Parasitic Antenna for Localization in Wireless Sensor Networks," 19th International Conference on Microwaves, Radar and Wireless Communications (MIKON 2012), vol. 1, pp.799-803, May 2012.

[6] E. Taillefer, C. Plapous, J. Cheng, K. Iigusa, and T. Ohira, "Reactancedomain MUSIC for ESPAR antennas (experiment)," in Proc. IEEE Wireless Commun. Netw. Conf., New Orleans, LA, USA, Mar. 2003, vol. 1, pp. 98-102.

[7] F. Viani, L. Lizzi, M. Donelli, D. Pregnolato, G. Oliveri, and A. Massa, "Exploitation of parasitic smart antennas in wireless sensor networks," J. Electromagn. Waves Appl., vol. 24, no. 7, pp. 993-1003, Jan. 2010.

[8] M. Rzymowski, P. Woznica and L. Kulas, "Single-Anchor Indoor Localization Using ESPAR Antenna," IEEE Antennas Wireless Propag. Lett., vol. 15, pp.1183-1186, 2016.

[9] Luis Brás, Nuno Borges Carvalho, Pedro Pinho, Lukasz Kulas, and Krzysztof Nyka, "A Review of Antennas for Indoor Positioning Systems," International Journal of Antennas and Propagation, vol. 2012, Article ID 953269, 14 pages, 2012.

[10] L. Kulas, "Direction-of-arrival estimation using an ESPAR antenna with simplified beam-steering," in Proc. 47th Euro. Microw. Conf., Nuremberg, Germany, 2017, pp. 296-299.

[11] L. Kulas, "RSS-based DoA Estimation Using ESPAR Antennas and Interpolated Radiation Patterns," IEEE Antennas Wireless Propag. Lett., vol. 17, pp.25-28, 2018.

[12] M. Groth and L. Kulas, "Accurate PPCC-Based DoA Estimation Using Multiple Calibration Planes for WSN Nodes Equipped with ESPAR Antennas," in Proc. 48th Euro. Microw. Conf. (EuMW 2018), Madrid, Spain, 2018, in press.

[13] S. Fudickar and M. Valentin, "Most accurate algorithms for RSS-based Wi-Fi indoor localisation," 2014 International Conference on Indoor Positioning and Indoor Navigation (IPIN), Busan, 2014, pp. 38-47.

[14] A. M. Tonello and D. Inserra, "Radio positioning based on DoA estimation: An implementation perspective," 2013 IEEE International Conference on Communications Workshops (ICC), Budapest, 2013, pp. 27-31.

[15] M. Passafiume, S. Maddio, M. Lucarelli and A. Cidronali, "An enhanced triangulation algorithm for a distributed RSSI-DoA positioning system," 2016 European Radar Conference (EuRAD), London, 2016, pp. 185-188. 